\title{
Are some people sensitive to mobile phone signals? Within participants double blind randomised provocation study
}

\author{
G James Rubin, Gareth Hahn, Brian S Everitt, Anthony J Cleare, Simon Wessely
}

\begin{abstract}
Objective To test whether people who report being sensitive to mobile phone signals have more symptoms when exposed to a pulsing mobile signal than when exposed to a sham signal or a non-pulsing signal.

Design Double blind, randomised, within participants

provocation study.

Setting Dedicated suite of offices at King's College London, between September 2003 and June 2005.

Participants 60 "sensitive" people who reported often getting headache-like symptoms within 20 minutes of using a global system for mobile communication (GSM) mobile phone and 60 "control" participants who did not report any such symptoms. Intervention Participants were exposed to three conditions: a $900 \mathrm{MHz}$ GSM mobile phone signal, a non-pulsing carrier wave signal, and a sham condition with no signal present. Each exposure lasted for 50 minutes.

Main outcome measures The principal outcome measure was headache severity assessed with a 0-100 visual analogue scale. Other outcomes included six other subjective symptoms and participants' ability to judge whether a signal was present. Results Headache severity increased during exposure and decreased immediately afterwards. However, no strong evidence was found of any difference between the conditions in terms of symptom severity. Nor did evidence of any differential effect of condition between the two groups exist. The proportion of sensitive participants who believed a signal was present during GSM exposure (60\%) was similar to the proportion who believed one was present during sham exposure (63\%).

Conclusions No evidence was found to indicate that people with self reported sensitivity to mobile phone signals are able to detect such signals or that they react to them with increased symptom severity. As sham exposure was sufficient to trigger severe symptoms in some participants, psychological factors may have an important role in causing this condition.

Trial registration ISRCTN81432775.
\end{abstract}

\section{Introduction}

The recent uptake of mobile phones has been accompanied by some concern about possible health risks. ${ }^{1}$ In the general population, the health effects most often attributed to mobile phone use are non-specific symptoms. Excluding sensations of mild warmth, the most commonly reported symptoms are headache, burning, dizziness, fatigue, and tingling. ${ }^{2}$ Mechanisms to explain these phenomena remain speculative, and although the pulsing nature of "global system for mobile communication" (GSM) signals has been suggested to be partly to blame, ${ }^{3}$ experiments that have exposed healthy adults to GSM signals under blind conditions have not found any significant effects on the reporting of symptoms. ${ }^{4}$

Whether a subgroup of people who are more sensitive to GSM exists remains unclear. Of particular interest are people who report symptoms almost every time they use a mobile phone. ${ }^{5}$ This phenomenon falls within the broader category of "electromagnetic sensitivity," a medically unexplained condition in which non-specific symptoms are reported after perceived exposure to any of a wide range of electrical devices, including mobile phones, visual display units, and power lines. The prevalence of self reported electromagnetic sensitivity in the United Kingdom is unknown, but community studies in Sweden and California put the figure at between $1.5 \%$ and $3 \% .{ }^{7}$ Provocation studies that have exposed people who report electromagnetic sensitivity to electromagnetic fields under blind conditions have so far failed to provide any good evidence linking the presence of electromagnetic fields to severity of symptoms. ${ }^{8}$ Several authors have therefore suggested that psychological mechanisms may be more relevant in causing the condition. ${ }^{9}$

We tested whether people with self reported sensitivity to GSM would experience greater headache severity after double blind exposure to a GSM signal than after exposure to a sham signal. Secondary outcomes included other symptoms and ability to discriminate GSM from sham signals. We also tested whether a pulsing signal resulted in greater reporting of symptoms than a non-pulsing signal.

\section{Methods \\ Study design}

In this within participants study, we exposed people who reported adverse reactions to mobile phone signals (sensitive group) or who did not report any such effects (control group) to three conditions: a signal mimicking that produced by a 900 MHz GSM mobile phone, an unpulsed continuous wave signal, and a sham exposure with no signal present. Our Clinical Trials Unit determined the order in which these conditions occurred for each participant on enrolment, by using a computerised random numbers generator and counter-balancing within blocks of six consecutive participants.

Exposures were double blind-that is, neither participants nor researchers were told which type of exposure was present in which testing session. The controls for our exposure equipment allowed for 256 possible settings, of which 15 had been randomly allocated to each condition. Only the Clinical Trials Unit knew which settings related to which exposure. For the first nine control participants and six sensitive participants (11.5\% of all participants), Clinical Trials Unit staff told researchers which 
setting to use on the morning of each exposure. Given the theoretical possibility that the meaning of a setting might eventually be inferred by observing several participants' reactions to it, for the remaining sessions Clinical Trials Unit staff entered the codes and then obscured them from the researchers with opaque tape.

\section{Participants}

To be eligible for the sensitive group, participants had to report often experiencing headache-like symptoms within 20 minutes of using a $900 \mathrm{MHz}$ GSM mobile phone. Participants who did not attribute any symptoms to mobile phone signals were eligible for the control group. We excluded people who were aged under 18 or over 75 , were pregnant, had a psychotic illness, were currently using antidepressants, or reported severe symptoms at baseline while in our testing room. We recruited participants through mailshots organised by an electromagnetic sensitivity support group, advertising by interested clinicians and by our funding body, posters in general practitioners' surgeries, adverts and articles in the press and specialist health publications, email circulars, and word of mouth.

\section{Exposures}

We generated exposures by using the standard GSM handset system used within the UK Mobile Telecommunications and Health Research programme. ${ }^{10}$ The antenna for this headband mounted system was positioned slightly above and behind the left ear and within a few millimetres of the participant's scalp. Both GSM and continuous wave conditions produced a target specific absorption rate adjacent to the antenna of $1.4 \mathrm{~W} / \mathrm{kg}$, with an uncertainty of $\pm 30 \%$. For the sham exposure, a continuous wave signal was generated to ensure that the system heated up to the same degree as the active exposures but was diverted to an internal load instead of being transmitted through the antenna; only minimal leakage of this signal occurred (specific absorption rate $<0.002 \mathrm{~W} / \mathrm{kg}$ ).

\section{Questionnaires}

We assessed severity of symptoms during exposure by using 100 mm visual analogue scales, ${ }^{11}$ anchored with the phrases "no sensation" and "worst possible sensation." These scales measured headaches; nausea; fatigue; dizziness; skin itching, tingling, or stinging; sensations of warmth or burning on skin; and eye pain or dryness.

We collected other data at baseline, consisting of demographics and current or previous mobile phone usage. We also asked participants to record the frequency with which they experienced 11 common symptoms after a mobile phone call (never, $25 \%$ of calls, $50 \%$ of calls, $75 \%$ of calls, every call). We asked participants in the sensitive group about duration of illness and symptoms, how near a mobile phone needed to be before they could detect it, whether they considered themselves to have "electrosensitivity or sensitivity to electromagnetic fields," whether they had sought treatment, and whether their sensitivity impaired their daily functioning. ${ }^{12}$

\section{Procedure}

We sent written information to people who contacted us and screened them for eligibility. We invited those who provided verbal consent to attend our unit for three mornings. We instructed participants not to take recreational drugs for one week before attending; not to drink alcohol for 24 hours beforehand; and not to drink more than one cup of tea or coffee, take painkillers, or undertake strenuous physical activity or anything psychologically stressful on the morning of each visit.
Sessions began with a 30 minute adjustment period. During this time in session one, participants provided informed written consent and completed the various demographic questionnaires. At the end of these adjustment periods, we asked participants to complete baseline visual analogue scale measures. The exposure equipment was then attached and switched on for 50 minutes. Participants completed further visual analogue scale measures after $5,15,30$, and 50 minutes. If a participant requested that an exposure be terminated early, visual analogue scales were administered immediately. All participants completed a final set of visual analogue scales 30 minutes after the end of each exposure. At this point we asked them to state whether they believed a signal had been present and their confidence about this (100 $\mathrm{mm}$ visual analogue scales from "complete guess" to " $100 \%$ certain"). At least 24 hours after each session we contacted participants and asked them whether they had experienced any visual analogue scale symptoms in the 24 hours since exposure. We ascertained a score of 0 (no sensation) to 10 (worst possible sensation) for any symptoms that were reported, and we categorised participants scoring 5 or more as having experienced a "definite" symptom.

All testing took place between September 2003 and June 2005 in two rooms within King's College London. The rooms, which were lit by two table lamps, were not shielded against outside electromagnetic fields.

\section{Sample size calculation}

We based our sample size calculation on our ability to detect a change in headache severity within the sensitive group after 50 minutes of GSM exposure, using a two way analysis of variance with one between participants factor (sensitive $v$ control) and one within participants factor (GSM $v$ continuous wave $v$ sham). On the basis of previous studies in healthy and electrosensitive participants, ${ }^{413}$ this analysis assumed that control participants would report a mean headache severity of 10 units in all three experimental conditions whereas sensitive participants would report a mean severity of 11.7 in the sham and continuous wave conditions, with standard deviations of 26.8. In the absence of any pre-existing data, we assumed correlations of $r=0.5$ between conditions and that any effect of GSM in the sensitive group would be moderate- that is, an effect size of 0.5 . Our calculation showed that to detect this effect as significant at the $5 \%$ level and with $80 \%$ power we would need 60 participants in each group. In practice, although these assumptions turned out to be reasonable, the nature of our data required us to adopt a different analytical strategy from that originally planned. As such, this calculation should be taken as indicative only.

\section{Analyses}

To analyse symptom severity over time, we used generalised estimating equations. ${ }^{14}$ This approach was needed to accommodate the extremely positively skewed distribution of each response variable and to allow the inclusion of a suitable correlation structure for the repeated measures of each response. These models also allowed us to take into account differing lengths of exposure for participants who requested that an exposure be terminated early. The specific generalised estimating equations model fitted to each response used $\log$ (symptom severity +1$)$ as the dependent variable, a gamma error distribution, and an exchangeable correlation structure. We used robust standard errors to judge the "significance" or otherwise of the explanatory variables included in the fitted models. ${ }^{14}$ 
Research

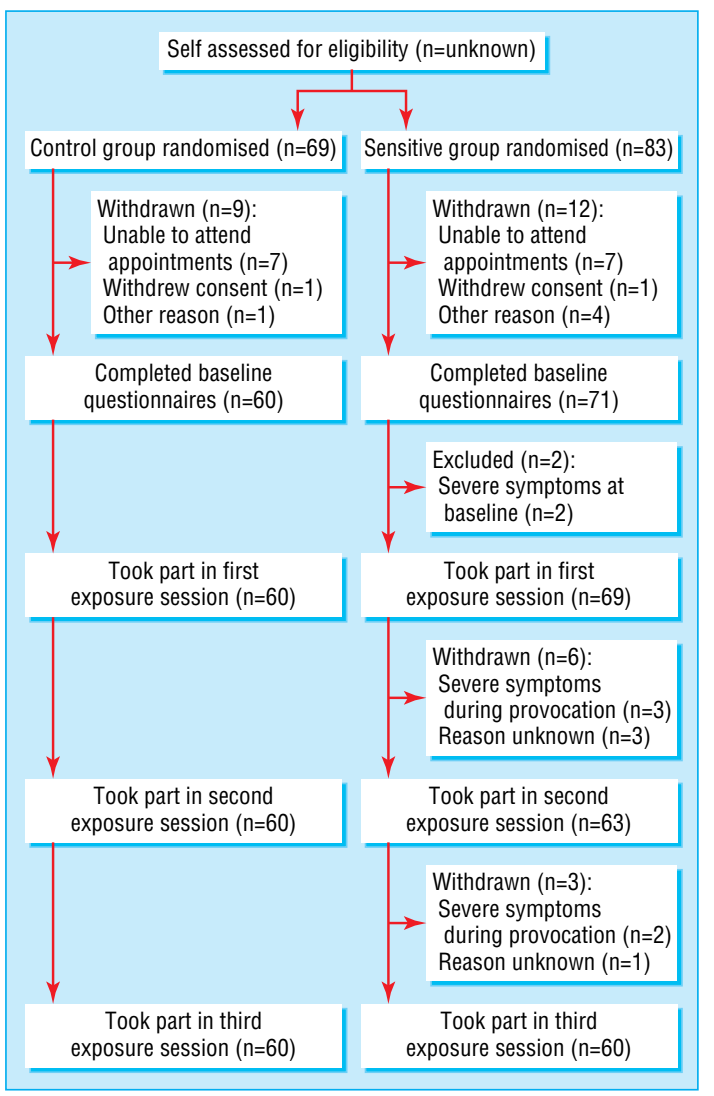

Fig 1 Study flow diagram

\section{Results}

We were contacted by 83 potential sensitive participants and 69 potential controls who met the inclusion criteria and provided verbal consent. Of these, 71 sensitive participants and 60 controls attended for their first testing session, and 60 in each group attended all three testing sessions and were included in our main generalised estimating equations analyses (fig 1). Table 1 shows demographic data for those participants who attended at least one session; the only substantive difference between the groups was a significantly higher proportion of sensitive participants from a professional or managerial background $\left(\chi^{2}=5.6\right.$, $\mathrm{P}=0.02)$. Restricting the demographic comparisons to participants who completed all three testing sessions did not alter these results.

For sensitive participants, the mean reported delay between beginning a call and onset of symptoms in everyday life was 6.5 (SD 6.5) minutes. For 48 people, symptoms usually resolved within two hours. All but one had been sensitive for at least a year (median 4 (interquartile range 2-5) years). Eighteen people reported that their sensitivity to mobile phones caused "definite" impairment or worse in at least one aspect of daily functioning, and 15 people reported having sought treatment for their condition. Thirteen people reported being sensitive to mobile phones at distances of one metre or more, and the same number reported having "electrosensitivity." Sensitive participants reported headache-like symptoms in a mean of $70.4 \%$ of calls. The next most common symptoms were skin warmth or burning (43.8\% of calls), difficulty concentrating $(30.0 \%)$, and dizziness $(20.8 \%)$. Very few control participants reported any symptoms in relation to mobile phone signals; the highest mean frequency was for skin warmth or burning $(2.9 \%)$.

\begin{tabular}{|c|c|c|c|}
\hline Variable & $\begin{array}{c}\text { Control group } \\
(n=60)\end{array}$ & $\begin{array}{l}\text { Sensitive group } \\
\qquad(n=71)\end{array}$ & $\begin{array}{c}P \text { value for } \\
\text { differences } \\
\text { between groups }\end{array}$ \\
\hline Mean (SD) age (years) & $33.5(10.2)$ & 37.1 (13.2) & 0.09 \\
\hline Sex (male:female) & $27: 33$ & $31: 40$ & 0.88 \\
\hline Ethnicity (white:other) & $45: 15$ & $56: 15$ & 0.60 \\
\hline $\begin{array}{l}\text { Marital status } \\
\text { (single:married/ } \\
\text { cohabiting:divorced/separated) }\end{array}$ & $39: 19: 2$ & $38: 30: 3$ & 0.41 \\
\hline $\begin{array}{l}\text { Employment status (in } \\
\text { work:unemployed:housewife/ } \\
\text { husband:student) }\end{array}$ & $30: 10: 2: 18$ & $42: 9: 3: 17$ & 0.71 \\
\hline $\begin{array}{l}\text { Socioeconomic status } \\
\text { (professional, managerial, or } \\
\text { intermediate:semiroutine, } \\
\text { routine, or student) }\end{array}$ & $31: 29$ & $51: 20$ & 0.02 \\
\hline $\begin{array}{l}\text { Educational level (secondary } \\
\text { education or lower:higher } \\
\text { education) }\end{array}$ & $18: 42$ & $26: 45$ & 0.42 \\
\hline $\begin{array}{l}\text { Weekly frequency of mobile } \\
\text { phone use }(<4 \text { times:4-12 } \\
\text { times:13+ times) })^{\star}\end{array}$ & $8: 25: 27$ & 17:22:32 & 0.23 \\
\hline $\begin{array}{l}\text { Typical length of call }(<5 \\
\text { minutes:5-15 minutes:16+ } \\
\text { minutes) }{ }^{*}\end{array}$ & $32: 22: 6$ & $44: 18: 9$ & 0.37 \\
\hline
\end{tabular}

Table 2 shows the results of fitting generalised estimating equation models to each response variable. The group $\times$ time interaction term was not needed in any model, so it does not appear in this table. Fitted models for all response variables showed highly significant effects for time (both linear and quadratic effects) and for baseline severity. We found no convincing evidence of an effect of condition or a condition $\times$ group effect for any of the symptoms. For headache, burning sensations, skin sensations, and eye pain we found evidence of a main group effect-sensitive participants reported greater severity. In terms of the original visual analogue scale units, this group effect for headache severity equated to an increase of 1.0 (95\% confidence interval 0.4 to 2.0) unit. Figure 2 shows the median headache severity by group for each exposure condition, and figure 3 illustrates the main effect of group on headache severity collapsed across conditions.

We also analysed the number of severe reactions seen in each condition, with a severe reaction defined as a participant requesting that an exposure be terminated early or withdrawing from the study entirely after an exposure. Twenty six such reactions occurred in the sensitive group ( 9 withdrawals; 17 early terminations), and none occurred in the control group. These reactions were equally distributed between $\operatorname{GSM}(n=7)$, continuous wave $(n=10)$, and sham $(n=9)$ conditions $\left(\chi^{2}=0.54\right.$, $\mathrm{P}=0.76$ ). Excluding data relating to the four participants whose reasons for withdrawal were not explicitly stated to us (see fig 1) did not affect these results (GSM 5, continuous wave 9, sham 8; $\chi^{2}=1.2, \mathrm{P}=0.55$ ).

We had next day follow-up results for all three sessions for 41 control participants and 49 sensitive participants. Cochran's $Q$ tests identified no significant differences in the number reporting at least one definite symptom after GSM, continuous wave, or sham exposures in either the control group (GSM 0/41, continuous wave $2 / 41$, sham $4 / 41 ; \mathrm{Q}=4.0, \mathrm{P}=0.14$ ) or the sensitive group (GSM 5/49, continuous wave 8/49, sham 4/49; $\mathrm{Q}=2.0, \mathrm{P}=0.37$ ).

Table 3 shows participants' assessments of whether a signal was present during provocation. The proportion who believed a signal was present during exposure to GSM $(60 \%$ of sensitive participants, $58 \%$ of controls) was slightly less than for the sham 
Table 2 Estimated regression coefficients (robust standard error) derived from generalised estimating equation models used to assess effects of group, exposure, duration of exposure, and baseline score on symptom severity

\begin{tabular}{|c|c|c|c|c|c|c|c|c|}
\hline Symptom & Baseline severity & $\begin{array}{l}\text { Duration (linear } \\
\text { function) }\end{array}$ & $\begin{array}{l}\text { Duration (quadratic } \\
\text { function) }\end{array}$ & $\begin{array}{c}\text { Sensitive } v \\
\text { control }\end{array}$ & Sham $v$ GSM & CW $v$ GSM & Group $\mathbf{x}$ (sham $v$ GSM) & Group $\mathbf{x}$ (CW $v$ GSM) \\
\hline Headache & $0.04(0.008)$ & $0.04(0.004)$ & $-0.0004(0.0004)$ & $0.7(0.2)$ & $0.07(0.1)$ & $-0.02(0.1)$ & $-0.08(0.2)$ & $0.2(0.2)$ \\
\hline Nausea & $0.02(0.05)$ & $0.006(0.001)$ & $-0.0002(0.00004)$ & $0.2(0.3)$ & $0.06(0.1)$ & $-0.2(0.1)$ & $0.1(0.4)$ & $0.3(0.3)$ \\
\hline Fatigue & $0.04(0.005)$ & $0.01(0.002)$ & $-0.0003(0.00005)$ & $0.2(0.2)$ & $-0.08(0.1)$ & $-0.2(0.1)$ & $-0.09(0.2)$ & $0.2(0.2)$ \\
\hline Dizziness & $0.05(0.02)$ & $0.007(0.001)$ & $-0.0003(0.00005)$ & $0.3(0.2)$ & $-0.2(0.1)$ & $-0.09(0.1)$ & $0.2(0.3)$ & $-0.01(0.3)$ \\
\hline$\overline{\text { Skin }}$ & $0.05(0.01)$ & $0.004(0.001)$ & $-0.0003(0.00005)$ & $0.5(0.2)$ & $-0.09(0.1)$ & $-0.1(0.1)$ & $0.1(0.2)$ & $0.3(0.2)$ \\
\hline Burning & $0.03(0.00005)$ & $0.007(0.001)$ & $-0.0007(0.0004)$ & $0.4(0.2)$ & $-0.05(0.1)$ & $-0.09(0.09)$ & $0.2(0.2)$ & $0.4(0.2)$ \\
\hline Eye pain & $0.05(0.008)$ & $0.007(0.001)$ & $-0.0003(0.00004)$ & $0.6(0.2)$ & $-0.04(0.1)$ & $0.2(0.1)$ & $-0.3(0.2)$ & $-0.08(0.2)$ \\
\hline
\end{tabular}

$\mathrm{CW}=$ continuous wave; $\mathrm{GSM}=$ global system for mobile communication.

In each model, the dependent variable used was log(symptom severity +1$)$.

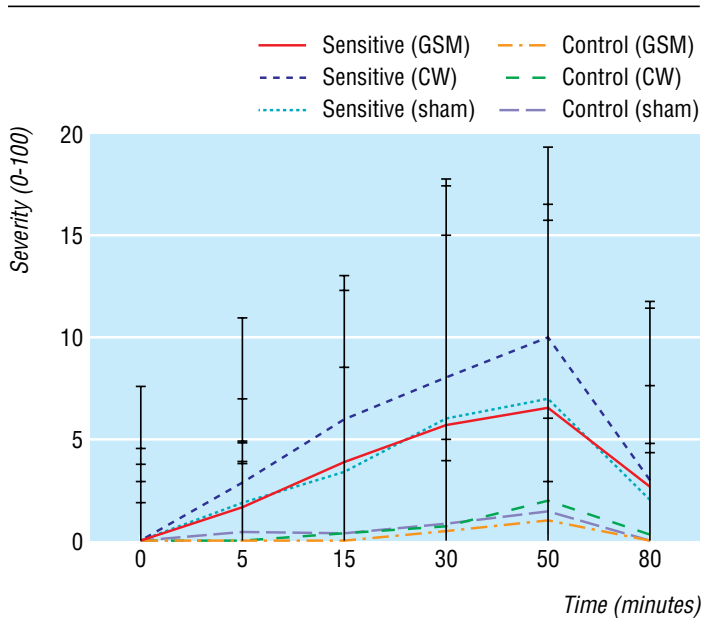

Fig 2 Median headache severity (error bars show interquartile range) during provocation with global system for mobile communication (GSM), continuous wave (CW), and sham exposures for sensitive and control participants. For clarity, graph does not include data relating to exposures that were terminated early, although these data were included in analyses exposure $(63 \%$ of sensitive participants, $68 \%$ of controls). Self reported confidence for these judgments did not differ greatly (table 3).

\section{Discussion}

We found no evidence to indicate that self reported sensitivity to $900 \mathrm{MHz}$ GSM mobile phone signals has a biological basis. Nor did we find any evidence to suggest that the pulsing nature of GSM contributes to these symptoms. These findings agree with the large majority of previous blind or double blind provocation studies for electromagnetic sensitivity, which have found no differences in the severity of symptoms elicited by active or sham exposure to electromagnetic fields. ${ }^{8}$

Did some inadequacy exist in our methods that might account for these "negative" findings? If it did, we are unaware of it. The exposure represented a relatively "worst case scenario" mobile phone call, using a high specific absorption rate and lasting almost eight times longer than the mean call length usually needed to trigger symptoms in our sensitive sample. Interference from participants' reactions to extraneous electromagnetic fields is also unlikely: after 30 minutes adjusting to our offices, only two

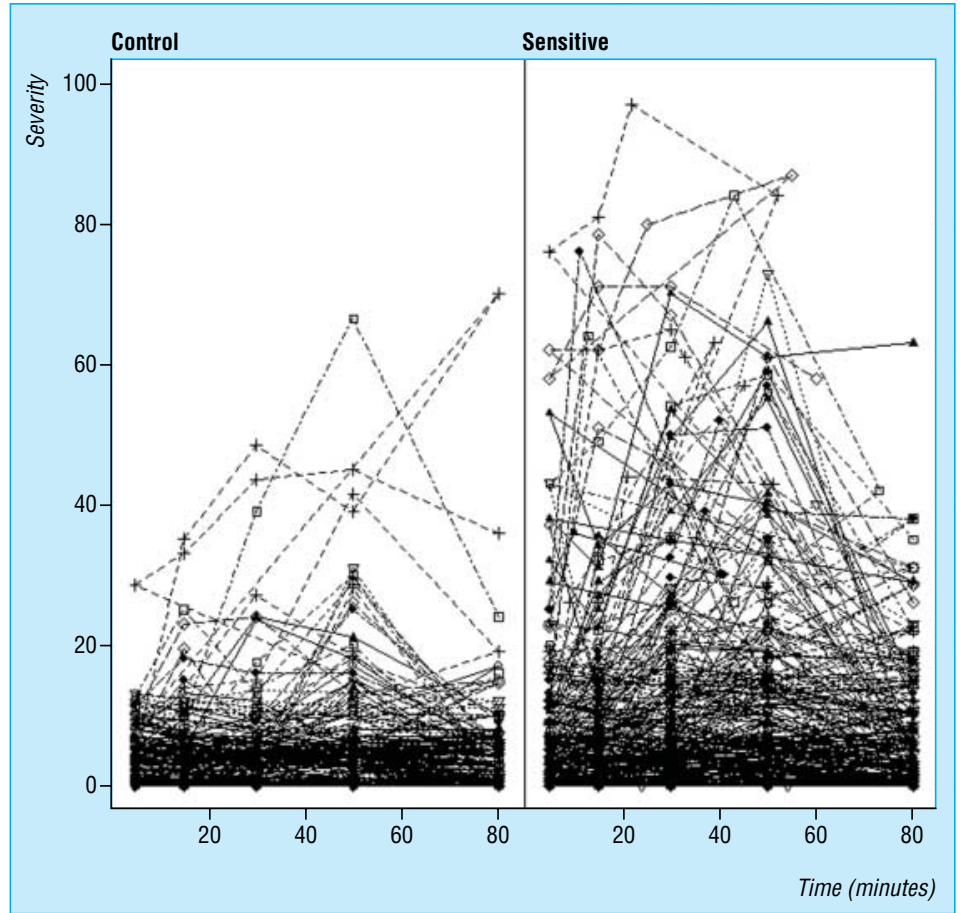

Fig 3 Headache severity over time for each participant, illustrating main effect of group on severity. Data for this figure have been collapsed across all three exposure conditions 
Table 3 Number of participants who believed a signal was present for each experimental condition and mean (SD) confidence (0-100) reported by participants for these "signal present" assessments

\begin{tabular}{|c|c|c|c|c|c|c|}
\hline \multirow{3}{*}{ Exposure } & \multirow{2}{*}{\multicolumn{2}{|c|}{ Controls }} & \multicolumn{4}{|c|}{ Sensitive participants } \\
\hline & & & \multicolumn{2}{|c|}{ Completed all three exposures } & \multicolumn{2}{|c|}{ Completed at least one exposure } \\
\hline & No & Confidence & No & Confidence & No & Confidence \\
\hline GSM & $35 / 60$ & $36.8(28.5)$ & $36 / 60$ & $58.6(30.8)$ & $41 / 65$ & $61.2(31.0)$ \\
\hline $\mathrm{CW}$ & $42 / 60$ & $39.7(33.0)$ & $41 / 60$ & $57.7(27.8)$ & $45 / 64$ & $57.8(28.9)$ \\
\hline Sham & $41 / 60$ & $43.9(31.9)$ & $38 / 60$ & $64.4(31.7)$ & $39 / 63$ & 64.0 (31.3) \\
\hline
\end{tabular}

$\mathrm{CW}=$ continuous wave; $\mathrm{GSM}=$ global system for mobile communication.

participants reported baseline symptoms that might have masked any effects of exposure, and both were excluded. Finally, as we were able to detect changes in symptom severity over time as highly significant, the sensitivity of our visual analogue scales and our statistical techniques do not seem to have had any shortcomings.

That symptom severity did increase during exposure is interesting. These symptoms were not trivial. Indeed, for some they were so severe that exposures had to be stopped early or the participants withdrew from the study. The confidence that sensitive participants had in their ability to discriminate active from sham signals also suggests that they experienced reactions similar to those encountered in real life, a finding also reported in previous provocation studies. ${ }^{8}$ That apparently realistic symptoms can be induced in provocation experiments, despite no differences being observed between active and sham conditions, suggests that the acute symptoms reported by sensitive people in everyday life may be the result of a nocebo phenomenon. Such phenomena have previously been observed in relation to a wide range of stimuli, ${ }^{15}$ including headaches induced by providing misleading information about the presence of electrical fields. ${ }^{16}$ The mechanisms governing nocebo effects need further study but seem to include conscious expectation of symptoms and the presence of negative affect, ${ }^{17}{ }^{18}$ factors that are likely to be present whenever people who perceive themselves to be sensitive to mobile phones have to make use of the technology.

In terms of their clinical implications, these results do not suggest that attempting to reduce exposure to mobile phone signals will be a useful strategy for patients who report sensitivity to them. Although such interventions might be actively sought by patients and may even produce a short term reduction in symptoms mediated by a placebo effect, ${ }^{19}$ in the longer term a danger exists that they will reinforce a patient's view of himself or herself as being sensitive to electromagnetic fields and put him or her at risk of developing symptoms associated with other electrical stimuli. Instead, it may be better to encourage such patients to test alternative non-electromagnetic field related explanations for their symptoms by using principles derived from cognitive behavioural therapy. ${ }^{9}$

We thank everybody who participated in this study, especially those in the sensitive group. We also thank Phil Chadwick from MCL-UK for supplying and calibrating the exposure equipment, and the staff from the Mental Health and Neurology Clinical Trials Unit at the Institute of Psychiatry for doing the randomisation and double blinding of the study.

Contributors: GJR had the original idea for the study and developed the study design with AJC, GH, and SW. GH and GJR did the testing. BSE analysed the symptom severity data; GJR did all other analyses. GJR wrote the first draft of the paper, and all authors contributed to further drafts. SW is the guarantor.

Funding: This study was funded by the Programme Management Committee (PMC) of the Mobile Telecommunications and Health Research (MTHR) programme (www.mthr.org.uk), an independent body set up to provide funding for research into the possible health effects of mobile telecommunications. The MTHR is itself jointly funded by the UK Department of Health and the mobile telecommunications industry. The
PMC contributed to the study design by proposing a reduction in overlap with other ongoing studies by focusing on symptom reporting, an increase in sample size, and an alteration in inclusion criteria to allow more highly sensitive people to participate. It had no role in the collection, analysis, or interpretation of the data, writing of the report, or decision to submit the paper for publication. The views expressed in this paper are those of the authors and not necessarily those of the funders.

Competing interests: None declared.

Ethical approval: The South London and Maudsley NHS Trust Research Ethics Committee granted approval for the study.

1 National Radiological Protection Board. Mobile phones and health 2004: report by the board of the NRPB. Documents of the NRPB 2004;15.

2 Oftedal G, Wilen J, Sandstrom M, Mild KH. Symptoms experienced in connection with mobile phone use. Occup Med 2000;50:237-45.

mobile phone use. Occup Med 2000;50:237-45.
Hyland GJ. Physics and biology of mobile telephony. Lancet 2000;356:1833-6.

4 Koivisto M, Haarala C, Krause CM, Revonsuo A, Laine M, Hamalainen H. GSM phone signal does not produce subjective symptoms. Bioelectromagnetics 2001;22:212-5.

Hocking B. Preliminary report: symptoms associated with mobile phone use. Occup Med 1998;48:357-60.

6 Hillert L, Berglind N, Arnetz BB, Bellander T. Prevalence of self-reported hypersensitivity to electric or magnetic fields in a population-based questionnaire survey. Scand J Work Environ Health 2002;28:33-41.

7 Levallois P, Neutra R, Lee G, Hristova L. Study of self-reported hypersensitivity to electromagnetic fields in California. Environ Health Perspect 2002;110(suppl 4):619-23.

Rubin GJ, Das Munshi J, Wessely S. Electromagnetic hypersensitivity: a systematic Rubin GJ, Das Munshi J, Wessely S. Electromagnetic hyp

Rubin G, Das Munshi J, Wessely S. A systematic review of treatments for electromagnetic hypersensitivity. Psychother Psychosom 2006;75:12-8.

10 MCL MTHR GSM and TETRA handset exposure systems for human volunteer studies. www.mcluk.org/MTHR_exposure_systems (accessed 23 Dec 2005).

11 McDowell I, Newell C. Visual analogue pain rating scales. In: McDowell I, Newell C, eds. Measuring health: a guide to rating scales and questionnaires. Oxford: Oxford University Press, 1996:341-6.

12 Mundt JC, Marks IM, Shear MK, Greist JH. The work and social adjustment scale: a simple measure of impairment in functioning. Br J Psychiatry 2002;180:461-4.

13 Andersson B, Berg M, Arnetz BB, Melin L, Langlet I, Liden S. A cognitive-behavioral treatment of patients suffering from 'electric hypersensitivity': subjective effects and reactions in a double-blind provocation study. J Occup Environ Med 1996;38:752-8.

\section{What is already known on this topic}

Non-specific symptoms such as headaches, tingling sensations, and fatigue are sometimes attributed to mobile phone use

No generally accepted mechanisms exist that might explain how mobile phone signals could cause such effects

A minority of people also report being particularly sensitive to mobile phones, experiencing symptoms almost every time they use one

\section{What this study adds}

The signals produced by $900 \mathrm{MHz}$ GSM mobile phones do not cause greater subjective symptoms than sham exposures in which no signal is present, even in people who report sensitivity to mobile phones

The symptoms reported by "sensitive" people may be the result of a nocebo effect and may be primarily psychological in origin 


\section{Research}

14 Everitt B. Modern medical statistics. London: Arnold, 2003.

15 Barsky AJ, Saintfort R, Rogers MP, Borus JF. Nonspecific medication side effects and the nocebo phenomenon. JAMA 2002;287:622-7.

16 Schweiger A, Parducci A. Nocebo: the psychologic induction of pain. Pavlov J Biol Sci 1981;16:140-3.

17 Benedetti F, Pollo A, Lopiano L, Lanotte M, Vighetti S, Rainero I. Conscious expectations and unconscious conditioning in analgesic, motor, and hormonal placebo nocebo responses. J Neurosci 2003;23:4315-23.

18 Petrie KJ, Moss-Morris R, Grey C, Shaw M. The relationship of negative affect and perceived sensitivity to symptom reporting following vaccination. $\mathrm{Br} J$ Health Psychol 2004;9:101-11.

19 Oftedal G, Nyvang A, Moen BE. Long-term effects on symptoms by reducing electric fields from visual display units. Scand J Work Environ Health 1999;25:415-21.

(Accepted 9 February 2006) doi $10.1136 / \mathrm{bmj} .38765 .519850 .55$

King's College London, Institute of Psychiatry, Department of Psychological Medicine, Section of General Hospital Psychiatry, Weston Education Centre (PO62), London SE5 9RJ

$\mathrm{G}$ James Rubin research fellow

Gareth Hahn senior research nurse

Anthony J Cleare senior lecturer

Simon Wessely professor of epidemiological and liaison psychiatry

King's College London, Institute of Psychiatry, Department of Biostatistics and Computing, London SE5 8AF

Brian S Everitt professor emeritus of biostatistic

Correspondence to: G J Rubin g.rubin@iop.kcl.ac.uk 